\title{
Piwil2: an IMPORTANT Regulator of Cancer Stem Cells in Breast Cancer Cell Line, MCF7-Piwil2
}

\author{
Fereshte Akbari ${ }^{1}$, Seyed Hamid Jamaldini ${ }^{1}$, Maryam Shahali ${ }^{2, *}$ \\ ${ }^{1}$ Department of Genetics, Tehran Medical Branch, Islamic Azad University, Tehran, \\ Iran \\ ${ }^{2}$ Pasteur Institute of Iran- 1- Department of Quality Control, Research and \\ Production Complex, Pasteur Institute of Iran, Tehran, Iran \\ ${ }^{*}$ Corresponding Author: Maryam Shahali, Pasteur Institute of Iran- -1 Department \\ of Quality Control, Research and Production Complex, Pasteur Institute of Iran, \\ Tehran, Iran.E-mail: maryam.shahali@gmail.com
}

DOI: $10.21859 /$ mci-supp-62

Keywords:

Piwil2

Cancer Stem Cells

Breast Cancer

\begin{abstract}
Introduction: Piwil2 belongs to Piwi protein subfamily, which functions in PIWI/piRNA pathway and plays crucial roles in gametogenesis in different organisms. Recent studies have demonstrated that PIWIL2 is ectopically expressed in tumor cell lines and various stages of different cancers, remarkably in cancer stem cell (CSCs). However, it is not clear whether Piwil2 has a causing role(s) in development of CSC populations or it is as a consequence of cancer. Thus, to further investigate the biological roles of Piwil2 in the process of tumorigenesis especially in cancer stem cells development, we established the MCF7-Piwil2 cell line stably expressing Piwil2.

Materials and Methods: MCF-7 cell line was transfected with a construct containing Piwil2 cDNA. Stable transfectants were selected and the effect of Piwil2 on its molecular behavior was investigated by semi-quantitative RT-PCR and qRT-PCR.

Results: The MCF7-Piwil2 cells demonstrated higher and lower expression of CSC biomarkers of CD44 and CD24, respectively, compared to MCF-7. We also examined CD133 expression and observed an increase in MCF7-Piwil2 cells. Totally, our molecular data were consistent with piwil2 reprogramming role in the emergence or prevalence of cancer stem cell population.

Conclusions: These observations may implicate piwil2 as an important regulator of cancer stem cells population in breast cancer. However, further complementary data such as the results of tumorigenic in vitro and in vivo assays of MCF-Piwil2 and its comparison with MCF-7 cells should be collected to fully confirm the role of piwil2 in the process of tumorigenesis.
\end{abstract}

